\title{
Specific Targeting of Ganglion Cell Sprouts Provides an Additional Mechanism for Restoring Peripheral Motor Circuits in Pelvic Ganglia after Spinal Nerve Damage
}

\author{
Mark E. Kepper and Janet R. Keast \\ Department of Physiology and Pharmacology, The University of Queensland, St. Lucia, Queensland, 4072, Australia
}

The pelvic ganglia contain both sympathetic and parasympathetic neurons and provide an interesting model in which to study the effects of a distributed spinal nerve lesion. Previous animal studies have suggested that after either lumbar or sacral nerve injury, some functional connections are restored between preganglionic and postganglionic neurons. It has been proposed that this is because of intact preganglionic axons sprouting collaterals to supply denervated ganglion cells. However, this has never been demonstrated, and our study has investigated whether the ganglion cells themselves contribute to axogenesis and restoration of peripheral circuitry. We have monitored the growth of axons from pelvic ganglion cells after lumbar or sacral nerve injury (partial decentralization), or a combination of the two (total decentralization). These new processes were distinguished from intact preganglionic terminals by their immunoreactivity for substances present only in pelvic ganglion neurons (vasoactive intestinal peptide, neuropeptide $\mathrm{Y}$, and tyrosine hydroxylase). The proportion of pelvic neurons surrounded by these immunostained fibers was then assessed. Complete removal of preganglionic terminals provides the biggest stimulus for growth of new axon processes (sprouts), which grow profusely within just a few days. These arise from each of the main chemical classes of pelvic neurons but grow at different rates and have different distributions. Importantly, some chemical classes of sprouts preferentially supply neurons of dissimilar histochemistry, suggesting the presence of very specific targeting mechanisms rather than random growth. These sprouts are transient, however, those formed after partial decentralization appear to be maintained. Moreover, after lesion of either lumbar or sacral spinal nerves, many sprouts arise from neurons with intact spinal connections and innervate neurons that have lost their preganglionic inputs. This provides a very different alternative mechanism to reestablish communication between preganglionic and postganglionic neurons. In conclusion, we have demonstrated a rapid and selective axogenesis within the pelvic ganglion after spinal nerve injury. This may allow the development of novel strategies by which autonomic nerve pathways can be experimentally manipulated, to facilitate more rapid return of appropriate peripheral reflex control.

Key words: autonomic ganglion; deafferentation; nerve injury; parasympathetic; pelvic reflexes; plasticity; sympathetic
Autonomic ganglia have been commonly exploited to examine synapse formation after nerve injury. Numerous studies have focused on the ability of regenerating spinal preganglionic axons to form functional connections with their target ganglion cells and have determined the time course, specificity, and physiological consequences of this synapse formation (for review, see Taxi and Eugène, 1995). Most of these experiments have been performed in paravertebral sympathetic ganglia and have demonstrated that many preganglionic axons eventually reestablish functional synapses, and do so with quite a remarkable degree of specificity.

A second type of axogenesis occurs much more rapidly, within just a few days of spinal nerve injury. After removal of preganglionic axons supplying sympathetic and parasympathetic ganglia, many axon processes grow "sprouts", presumably from denervated ganglion cells (Quillam and Tamarind, 1972; Sargent and Dennis, 1977; Dail and Evan, 1978; Yokota and Burnstock, 1983; Ramsay and Matthews, 1985; Dail and Minorsky, 1986; Johnson, 1988; Minorsky and Dail, 1993; Dail et al., 1997). This assumption

\footnotetext{
Received April 7, 1998; revised June 17, 1998; accepted July 14, 1998.

This work was supported by the National Health and Medical Research Council of Australia. Correspondence should be addressed to Dr. Janet Keast, Department of Physiology and Pharmacology, The University of Queensland, St. Lucia, Queensland, 4072, Australia.

Copyright (ㄷ) 1998 Society for Neuroscience $\quad 0270-6474 / 98 / 187987-09 \$ 05.00 / 0$
}

is based on the chemical similarity between sprouts and the ganglion cells, as well as the lack of other possible sources; notably, they have inappropriate chemistry and appear too soon to be regenerated processes of spinal axons. These sprouts form varicose terminals around neighboring ganglion cells and in some cases have been shown to form morphological and functional synapses (Quillam and Tamarind, 1972; Sargent and Dennis, 1977; Yokota and Burnstock, 1983; Ramsay and Matthews, 1985; Johnson, 1988). The function of these sprouts and the mechanisms underlying their formation are not known.

A particularly interesting place in which to investigate the consequences of spinal nerve injury is the pelvic plexus, which consists of ganglion cells that supply the urogenital organs and lower bowel. This plexus is a mixture of sympathetic and parasympathetic neurons and receives spinal inputs from the lumbar and sacral levels of the spinal cord (Keast, 1995b). It is well known that damage to lumbar spinal outflow leads to a failure of reflexes such as emission, whereas sacral nerve damage causes loss of the micturition and erection reflexes (Keast, 1995a). However, there is intriguing evidence from animal studies that the peripheral motor component of these reflex circuits can be reestablished and that this does not involve regeneration of damaged spinal axons. For example, between 3 and $7 \mathrm{~d}$ after pelvic (sacral) nerve transection in rats, a substantial penile erection reflex can be elicited by hypogastric (lumbar) nerve stimulation, whereas in 
intact animals this elicits only a very small response (Dail et al., 1989). Similar success in restoration of ganglionic connectivity has also been seen for the micturition reflex (de Groat and Kawatani, 1989) and contraction of the vas deferens (Kihara et al., 1996) after damage to sacral and lumbar spinal nerves, respectively.

The popular interpretation of these observations has been that after incomplete lesion of spinal nerves, undamaged preganglionic axons grow collateral processes to innervate those ganglion cells that have lost their spinal connections (Dail et al., 1989; de Groat and Kawatani, 1989; Kihara et al., 1996). However this axogenesis has never been directly demonstrated, and a second possibility exists. In pelvic ganglia, as in other autonomic ganglia, sprouts grow rapidly after removal of preganglionic inputs, and their likely source is the ganglion cells (Dail and Evan, 1978; Yokota and Burnstock, 1983; Dail and Minorsky, 1986; Minorsky and Dail, 1993; Dail et al., 1997). We propose that after partial decentralization these sprouts will originate from ganglion cells that have retained their spinal connections and, as such, constitute a quite different mechanism by which signaling between preganglionic and postganglionic neurons can be reestablished.

The present study has exploited the relative simplicity of the rat pelvic plexus, consisting of the paired major pelvic ganglia, to investigate the anatomical changes that occur in response to removal of some or all spinal inputs. We have tested two hypotheses. The first is that after either partial or total preganglionic denervation, sprouts are formed by and target specific types of neurons, i.e., they do not grow or associate randomly. The second hypothesis is that after partial removal of spinal inputs to the pelvic ganglion, sprouts are formed by postganglionic neurons with intact spinal connections, and they selectively target neurons that have been denervated. Analysis of the chemical profile of sprouts, the types of neurons with which they are associated, and the time course of sprout formation after different types of nerve injury has allowed comparison with known physiological changes after spinal damage. Our results have supported both hypotheses. They have shown that there is very specific targeting of sprouts within this mixed population of neurons. They are also the first to show that restoration of peripheral reflex pathways after spinal damage occurs at least in part because of postganglionic neuron sprouting.

\section{MATERIALS AND METHODS}

Seventy-four male Wistar rats (138-200 gm) were used. All surgical procedures were performed under general anesthesia with sodium pentobarbitone $(45 \mathrm{mg} / \mathrm{kg}$, i.p.).

Nerve lesions and immunostaining of ganglion cells and sprouts. Two types of bilateral nerve lesion were performed. In some animals, both the lumbar and sacral preganglionic axons supplying the major pelvic ganglia were transected (total decentralization), whereas in others only one group was lesioned (partial decentralization). Lumbar and sacral preganglionic axons were lesioned by transection of the hypogastric (HGN) and pelvic nerves (PVN), respectively. After accessing the ganglia via a ventral midline abdominal incision, the HGN and PVN were separated from their surrounding connective tissue with fine forceps and transected 3-5 $\mathrm{mm}$ from each ganglion. A 1-2 $\mathrm{mm}$ portion of each proximal nerve stump was removed to ensure that regeneration of preganglionic fibers could not occur within the time period analyzed. For PVN transection, extra care was taken not to damage large blood vessels that often travel with the nerves. After PVN transection a "neurogenic bladder" resulted from disruption of the micturition reflex, and twice daily the bladder was manually emptied by applying gentle pressure to the lower abdomen. No obvious return of normal bladder reflex function appeared after nerve injury.

Total decentralization was performed on 26 animals that were allowed to survive for either $1,2,4,8$, or $12 \mathrm{~d}$ ( $\pm 1 \mathrm{hr}$; four animals per group).
A further four animals served as unoperated controls (i.e., $0 \mathrm{~d}$ ). In the partial decentralization experiments, ganglia were analyzed 4, 8, and $12 \mathrm{~d}$ after either HGN or PVN lesion (four animals per group). Sham lesions $(n=2)$ involved separation of the HGN and PVN from surrounding connective tissue and blood vessels, without nerve transection, and a survival period of $8 \mathrm{~d}$.

After the allocated time, each animal was reanaesthetized, and the pelvic ganglia were removed. Ganglia were immediately placed in a dish lined with silicon polymer and briefly rinsed in $0.1 \mathrm{M} \mathrm{PBS,} \mathrm{pH}$ 7.2. Pins were placed through the tips of each nerve to maintain the ganglion flat and in its typical orientation during fixation. The tissues were then stored at $4{ }^{\circ} \mathrm{C}$ overnight in Zamboni's fixative (a mixture of $0.2 \%$ picric acid and $2 \%$ formaldehyde, buffered with $0.1 \mathrm{~m}$ phosphate buffer, $\mathrm{pH} 7.4$ ), followed by washes in dimethylsulfoxide (three times for $10 \mathrm{~min}$ each) and PBS (three times for $10 \mathrm{~min}$ each).

Before sectioning, the ganglia were cryoprotected with PBS containing $30 \%$ sucrose. Serial cryostat sections $(10 \mu \mathrm{m})$ were divided between three sets of slides, and immunolabeling for TH, NPY, and VIP was performed so that every third section was stained for the same substance. These substances label virtually all of the pelvic ganglion neurons, which are known to consist of three major chemical types: (1) parasympathetic cholinergic NPY neurons, (2) sympathetic noradrenergic NPY neurons, and (3) cholinergic VIP neurons, of which most are parasympathetic and approximately one quarter are sympathetic (i.e., have lumbar spinal inputs; Keast, 1995b). Specific, characterized antisera were used, as described previously (Kepper and Keast, 1995). Sections were incubated at room temperature for 18-24 hr with primary antisera raised in rabbits against VIP (1:2000, Incstar, Stillwater, MN), NPY (1:3000, Incstar), and TH (1:1000, Eugene Tech, Ridgefield Park, NJ). Commercially obtained secondary antisera were raised in donkeys against rabbit Igs and conjugated to the fluorophore Cy-3 (Jackson Immunochemicals, West Grove, PA). They were used at a dilution of 1:1000 and incubated for $2 \mathrm{hr}$ at room temperature.

Immunostained structures from the largest section of each ganglion were viewed using the N2 filter of a Leitz microscope. The total number of neurons in the section was determined by counting autofluorescent, nucleated neuronal profiles viewed with the A filter. Any neuron with a total of at least $75 \%$ of its soma profile surrounded by one or more varicose immunoreactive axons was defined as being supplied by a sprout "basket". This value was chosen to restrict our analyses to terminals that were very likely to be specifically associated with each neuron of interest. In many cases by focusing through the section, neurons supplied by sprouts appeared to have much of their surface covered by a plexus of beaded varicosities (Fig. 1). The histochemical class of each neuron and its associated basket was also recorded to determine whether sprouts supplied neurons with a similar histochemistry.

Retrograde labeling of neurons supplying different pelvic organs. In 12 animals, total decentralization was performed as described above. Under the same period of anesthesia, small volumes (2-4 $\mu \mathrm{l})$ of the retrograde tracers fluorogold $(\mathrm{FG})$ or fast blue (FB) were injected into a single pelvic organ, as described in previous studies (Keast and de Groat, 1989). In six of these animals, FB and FG were injected into two different organs (colon and prostate gland or seminal vesicles and vas deferens). Neurons innervating the penis or bladder were studied in separate animals, using FB or FG, respectively. Three animals were used for each organ injection.

After $4 \mathrm{~d}$, pelvic ganglia were removed and processed for immunolabeling as described above. This time was chosen because pilot studies showed that this is the earliest after total decentralization when significant numbers of baskets exist. The proportions of FG- or FB-labeled nucleated profiles supplied by TH, NPY, or VIP sprout baskets were determined in all ganglion sections by alternate viewing through the $\mathrm{A}$ filter for retrograde tracers and N2 filters for immunostaining.

Visualization of intact preganglionic terminals and sprouts after partial decentralization. In this series of experiments, the PVN or HGN were transected in twelve animals (four for each type of nerve transection). Twelve days after surgery, pelvic ganglia were removed and processed for immunostaining. The same primary antisera were used as described above, with the addition to each of antiserum raised against the synaptic vesicle protein, synaptophysin (1:50, Dako, Carpinteria, CA). In previous studies this has been shown to brightly label all preganglionic axon terminals in the rat pelvic ganglia (Keast, 1995b). Neurons were therefore considered to be denervated if they were not supplied 

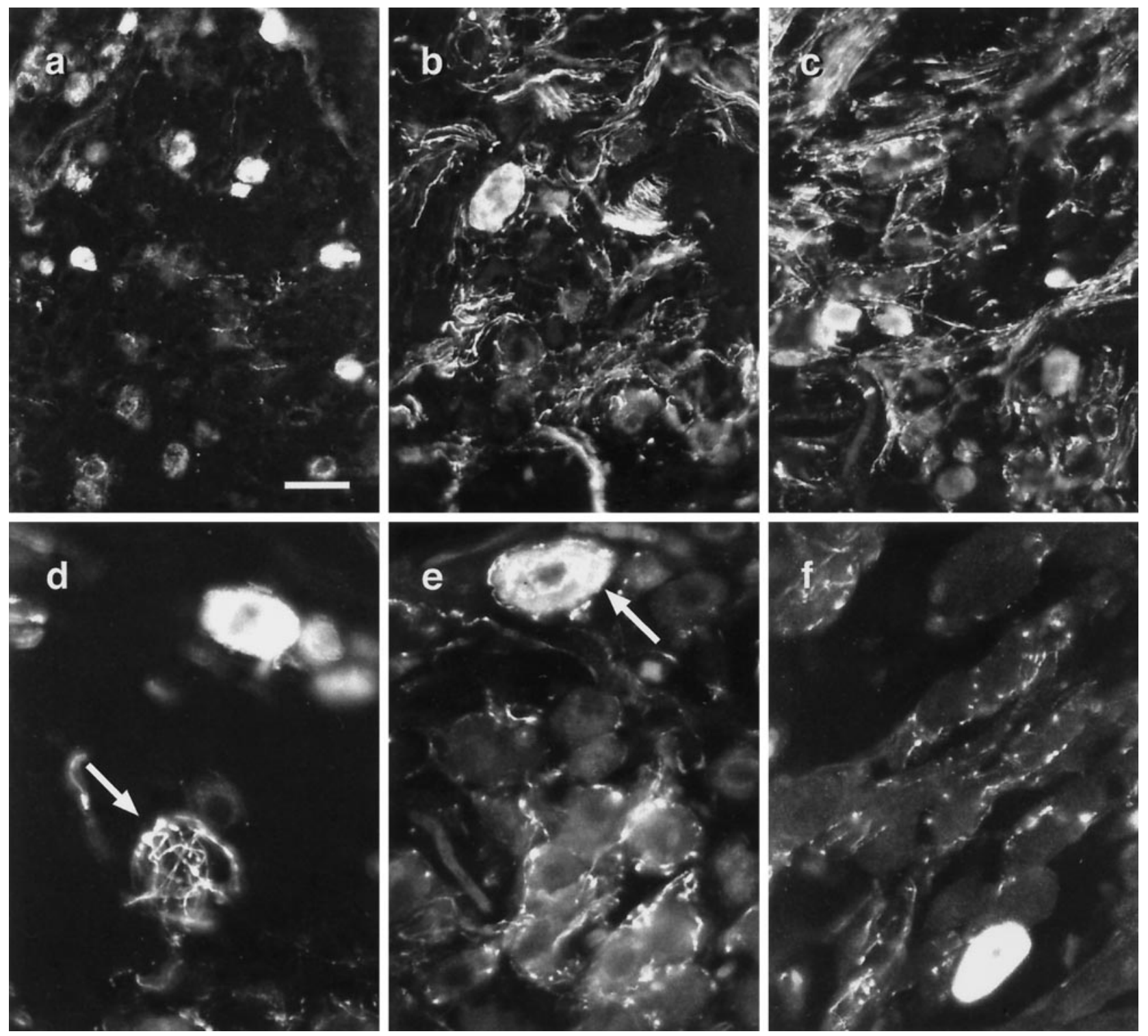

Figure 1. Sprout formation in pelvic ganglia after total decentralization. VIP sprout formation in the dorsal region of the pelvic ganglion in $(a)$ a control (intact) animal and after total decentralization, $(b) 2 \mathrm{~d}$, and $(c) 4 \mathrm{~d}$ after nerve transections. $d-f$, Baskets formed $8 \mathrm{~d}$ after total decentralization are shown at higher magnification. $d$, A VIP basket (arrow) encapsulates a VIP-negative neuron in the ventral region of the ganglion. $e$, An NPY basket is associated with an NPY neuron (arrow), whereas a nearby group of NPY-negative neurons is also surrounded by NPY baskets. $e$, TH baskets are always associated with TH-negative neurons. Scale bar applies to all micrographs: $a, 50 \mu \mathrm{m}(a-c) ; 25 \mu \mathrm{m}(d-f)$.

by bright synaptophysin-positive varicosities (Keast, 1995b). Synaptophysin immunoreactivity was visualized with a secondary antibody raised in donkey against mouse $\mathrm{IgG}$ and conjugated to fluorescein isothiocyanate (1:50; Jackson ImmunoResearch, West Grove, PA) and viewed under the L3 filter. The number of neurons supplied by TH, NPY, or VIP sprout baskets were counted in each ganglion section, and the proportion of baskets supplying denervated neurons was determined.

Statistics. All data are expressed as mean \pm SE. For TH, NPY, or VIP, the number of sprout baskets was compared between total and partial decentralization and at different times using one-way and two-way ANOVA, as appropriate. For post hoc comparison of individual groups, either a Tukey-Kramer (unequal $n$ values) or Student's Newman-Keuls test (equal $n$ values) was used. One-way ANOVA was used to determine whether there were any differences between the supply of sprout baskets to neurons innervating different pelvic organs after total decentralization. In all cases $p<0.05$ was considered a significant difference between groups.

\section{RESULTS}

Total decentralization causes rapid axogenesis from the three major types of pelvic neurons

The distribution of TH, NPY, and VIP within the pelvic ganglion of unoperated animals resembled previously published descriptions (Keast and de Groat, 1989; Keast, 1995b). Many somata and smooth (presumably postganglionic) axons were stained (Fig. 1a), in addition to smooth and varicose axons within numerous postganglionic nerve bundles. TH-immunoreactive small intensely fluorescent (SIF) cells were also present. In a few instances, ganglion cells were encapsulated by varicose VIP or NPY axons, however, this occurred for $<1 \%$ of neurons. These were consistently located in the dorsal region of the ganglion near the entrance of the pelvic and cavernous nerves. Within $2 \mathrm{~d}$ of total decentralization, there was a rapid formation of many axons (i.e., 


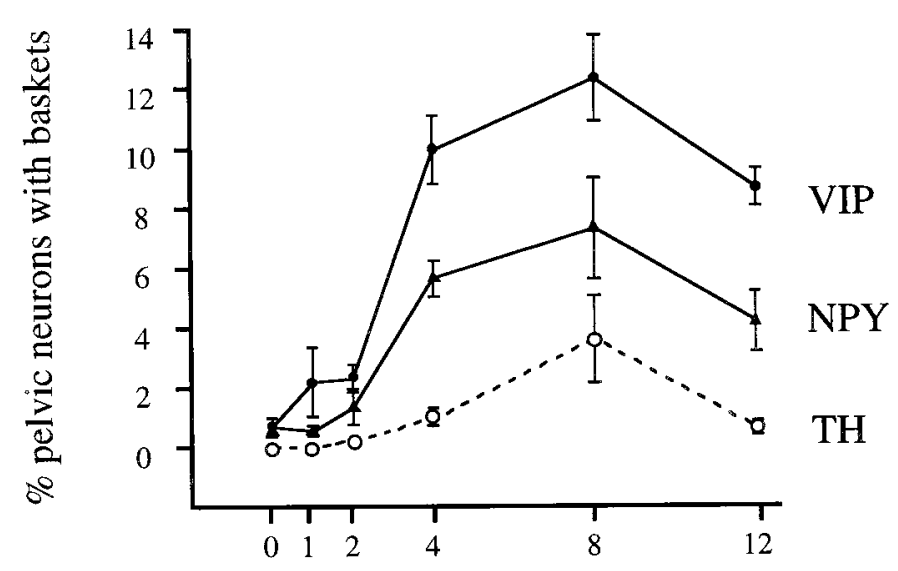

Days after total decentralisation

Figure 2. Time course of basket formation after total decentralization of the pelvic ganglion. Data are expressed as mean $\pm \operatorname{SE}(n=4$ animals for each time point). Where error bars are not shown, these are smaller than the symbols.

sprouts) that could be demonstrated with VIP (Fig. 1b) and NPY immunolabeling. At this time, axogenesis was isolated to the dorsal pole of the ganglion, and many axons in this region appeared to be closely associated with neuron somata. However very few were classified as "baskets", because they did not satisfy the criteria of association with at least $75 \%$ of the soma profile circumference. Four days after total decentralization, there was a substantial increase in sprout and basket formation from VIP and NPY neurons, which was maintained or slightly increased by $8 \mathrm{~d}$ (Figs. 1c-e, 2). However, TH baskets were not produced above control levels until $8 \mathrm{~d}$ after total decentralization (Figs. 1f, 2). In sham-operated animals, the proportion of neurons supplied with baskets did not differ significantly from control levels (data not shown). Somata immunoreactive for the three markers were similarly distributed in the control and decentralized ganglia, although VIP immunofluorescence was brighter in a few neurons from 2 to $12 \mathrm{~d}$ after decentralization. Previous studies using similar lesions to identify sympathetic and parasympathetic neurons supplying various pelvic organs have shown that there is very little or no change in immunohistochemical phenotype of pelvic neurons after either type of nerve lesion (Kepper and Keast, 1995, 1997; Luckensmeyer and Keast, 1995).

There were considerable differences between the three different sprout types in the numbers and structure of their baskets. First, VIP baskets were more prevalent than NPY baskets, whereas TH baskets were relatively sparse (Fig. 2). Each of the three basket populations reached a maximum number by $8 \mathrm{~d}$, but then decreased $12 \mathrm{~d}$ after nerve transections. This was most apparent for $\mathrm{TH}$ baskets that had returned to control levels by this time. Second, the structure of VIP and TH baskets differed. VIP baskets typically were comprised of numerous axons with many varicosities (Fig. 1d), whereas TH baskets were usually formed of a more sparse network of axons, each with only few varicosities (Fig. 1f). NPY baskets were more variable in structure and included both dense and sparse networks of axons (Fig. 1e). As numerous baskets of each type were examined (3371425), these differences are likely to represent genuine differences in structure of the three types.

\section{Lumbar and sacral decentralization trigger different types of axogenesis}

In comparison with total decentralization, partial decentralization caused very different effects on axogenesis. Although the structure of baskets was comparable after the two types of nerve injury, the time course and extent of basket formation differed substantially (Fig. 3). The most noticeable difference is that partial decentralization initiated far less axogenesis, as demonstrated by the decreased number of baskets and the longer latency of basket formation. Furthermore, in all but one time point and for one substance (TH, $12 \mathrm{~d}$ after decentralization), the sum of baskets produced after individual nerve transections was exceeded by the number of baskets formed after total decentralization $(p<0.05)$.

One of the most important observations resulting from partial decentralization was that each type of lesion initiated a different type of axogenesis. In particular, TH and NPY baskets increased above control levels only after parasympathetic decentralization. Because all noradrenergic pelvic neurons are sympathetic (i.e., receive lumbar spinal inputs) and contain NPY (Keast, 1991, 1995 b), these sprouts must have arisen from neurons with intact spinal connections. Therefore, parasympathetic decentralization provides the optimal stimulus for sprout formation from sympathetic noradrenergic neurons.

In contrast to TH and NPY, both types of partial decentralization initiated formation of similar numbers of VIP baskets within $8 \mathrm{~d}$ of either nerve lesion. Therefore, there is no evidence for either parasympathetic or sympathetic decentralization being the preferred stimulus for VIP sprout formation. However, VIP neurons comprise a mixture of sympathetic and parasympathetic types, and it was not possible to discriminate between them in the present study. Our results do not preclude the possibility of sympathetic VIP neurons forming sprouts only after parasympathetic nerve lesion, or vice versa.

\section{Sprout baskets preferentially supply ganglion cells that have a dissimilar histochemistry}

Ganglia analyzed in the earlier sections of the study were also used to compare the neurochemistry of baskets and their associated neurons. Generally, baskets were associated with neurons that had a dissimilar histochemistry (Fig. 4). This indicates that baskets did not supply neurons randomly, but they specifically targeted (or avoided) certain groups of cells. This was particularly striking for $\mathrm{TH}$ baskets, in which only one of the $16,609 \mathrm{TH}$ neurons analyzed was supplied by a TH-positive basket $8 \mathrm{~d}$ after total decentralization. Typically, $\mathrm{TH}$ sprouts and baskets were located in areas sparsely populated by $\mathrm{TH}$-immunoreactive neurons (Fig. 1f). Similarly, at most time points VIP baskets preferentially supplied VIP-negative neurons (Figs. 1d, 4). In contrast, the specificity of NPY baskets was less apparent, and up to $45 \%$ of these baskets were associated with neurons of a similar histochemistry after either sympathetic or total decentralization (Figs. 1e, 4). However, NPY neurons include two chemical classes, noradrenergic and cholinergic, and we did not discriminate between these in this study. Our results therefore do not preclude histochemical specificity of NPY sprout targeting.

\section{Neurons with different peripheral targets are similarly supplied by sprouts after total decentralization}

In both intact and decentralized ganglia, the distribution of retrogradely labeled pelvic neurons resembled previous descriptions (Keast and de Groat, 1989; Keast, 1992; Kepper and Keast, 

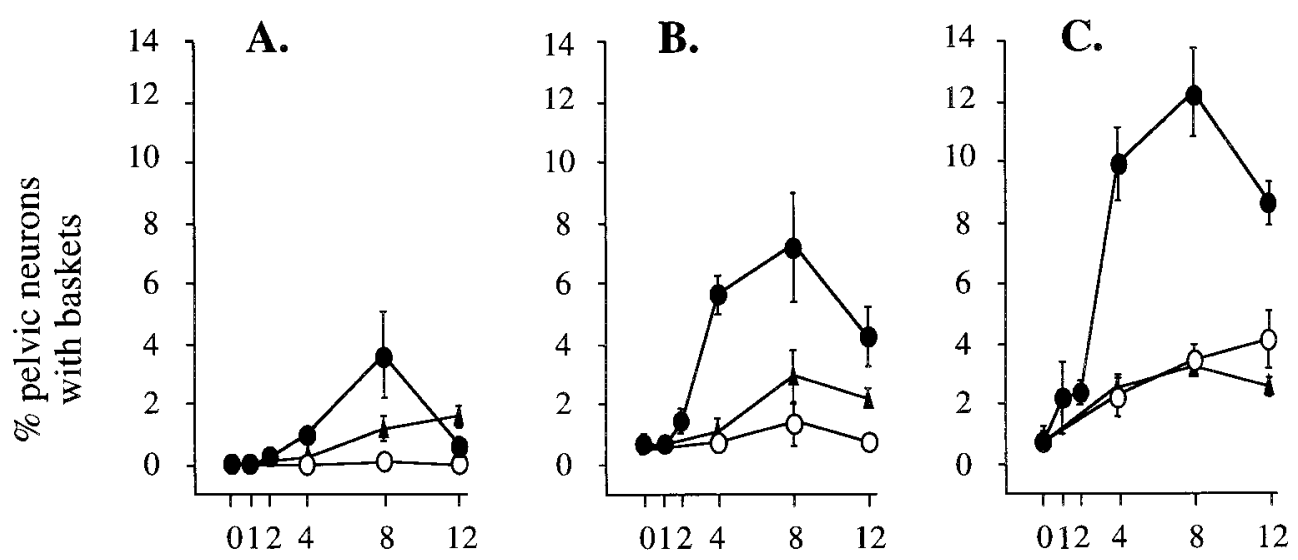

Days after decentralisation

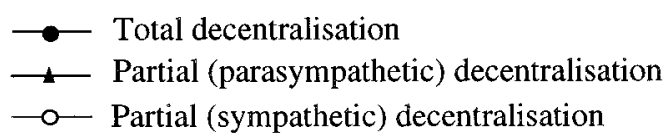

Figure 3. Time course of basket formation after total or partial decentralization of the pelvic ganglion. Time course of $(A)$ TH, $(B)$ NPY, or $(C)$ VIP basket formation from totally decentralized ganglia $0,1,2,4,8$, and $12 \mathrm{~d}$ after nerve lesions (data are the same as those shown in Fig. 2). Partially decentralized ganglia were only examined $0,4,8$, and $12 \mathrm{~d}$ after nerve transection. Data are expressed as mean \pm SE ( $n=4$ animals for each time point). Where error bars are not shown, these are smaller than the symbols. From each animal, 438-1528 neurons were examined for the presence of baskets.

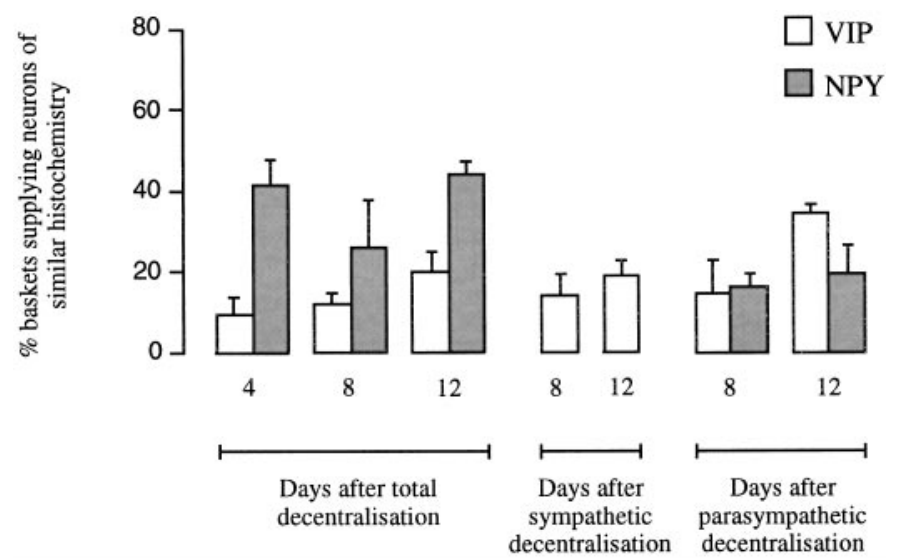

Figure 4. Histochemical features of pelvic neurons and their associated baskets that have formed after partial or total decentralization. $\mathrm{TH}$ baskets do not supply TH neurons, and NPY baskets are not formed above control values 8 or $12 \mathrm{~d}$ after sympathetic decentralization. Data are expressed as mean $\pm \mathrm{SE}$, at which four animals were analyzed for each point.

1995, 1997). The proportion of labeled neurons supplied by baskets after total decentralization was calculated to determine whether neurons supplying particular organs were preferentially targeted (Figs. 5, 6). For neurons supplying each organ, the relative prevalence of each basket type was similar to that found in the whole population of pelvic neurons (i.e., VIP sprouts were the most prevalent and $\mathrm{TH}$ baskets were sparse). Although there did appear to be some differences between organs (e.g., the proportion of VIP baskets supplying bladder- and penisprojecting neurons), they did not reach statistical significance (Fig. 6). Furthermore, regardless of the organ of innervation, VIP baskets were typically associated with neurons of a dissimilar
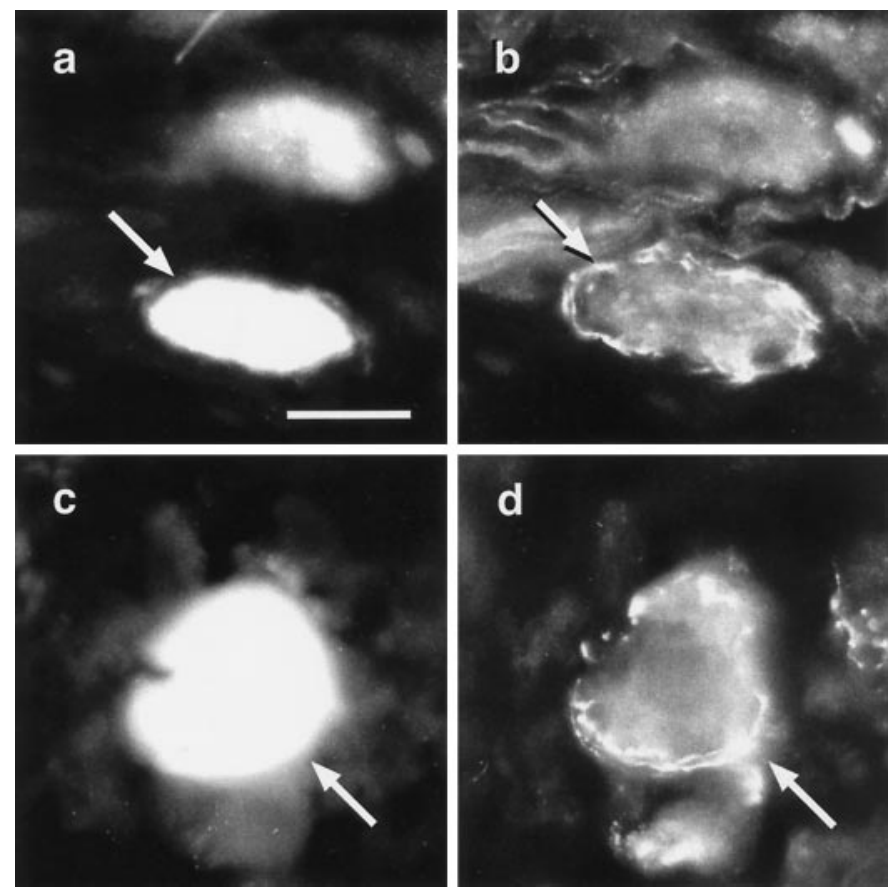

Figure 5. Association of sprout baskets with neurons of known target organs. $a$, A fluorogold-labeled pelvic neuron supplying the prostate gland (arrow) is encapsulated by an NPY basket (b) $4 \mathrm{~d}$ after total decentralization. $c$, A fast blue-labeled neuron innervating the vas deferens (arrow) is supplied by a VIP basket $(d)$. Scale bar: $a, 25 \mu \mathrm{m}$ (applies to all micrographs).

histochemistry, whereas NPY baskets were evenly distributed between NPY-positive and NPY-negative neurons (Table 1). The only exception to this was for penis-projecting neurons, in which 


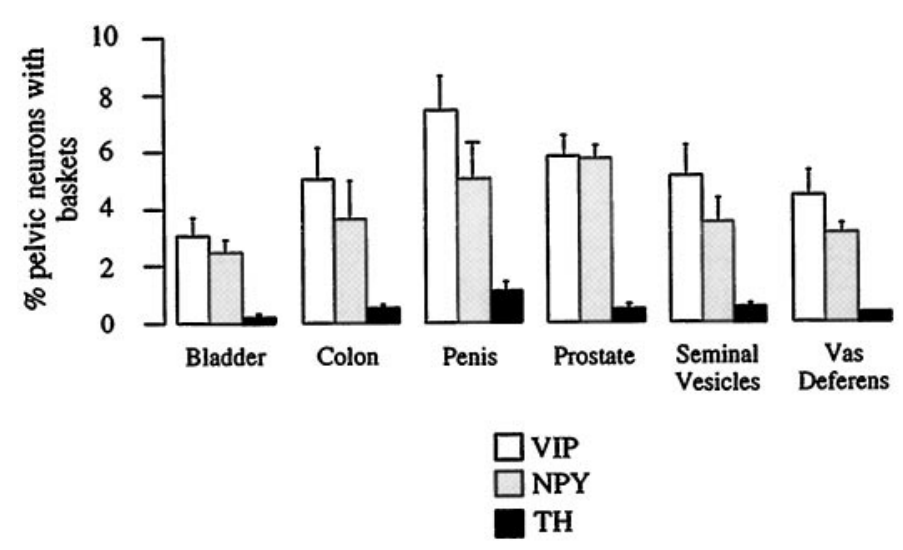

Figure 6. Target neurons of sprout baskets formed after total decentralization of the pelvic ganglia. The percentage of pelvic ganglion cells retrogradely labeled from each organ that are supplied by baskets $4 \mathrm{~d}$ after total decentralization. For each type of sprout basket (TH, NPY, or VIP), no statistical differences were associated with pelvic neurons projecting to any of the pelvic organs. From each animal, between 154-1755 retrogradely labeled neurons were analyzed $(n=3$ animals for each point).

$>90 \%$ of VIP baskets supplied VIP-positive neurons (Table 1). However, penile neurons are unique among the group because they are comprised almost exclusively of VIP neurons (Dail et al., 1983; Keast and de Groat, 1989); therefore, even if only a small proportion of VIP sprouts target penile neurons, they will necessarily innervate neurons of similar histochemistry.

\section{After partial decentralization, sprout baskets supply denervated neurons}

After all types of decentralization, neurons were examined for possible colocalization of intact preganglionic terminals and baskets. Intact spinal innervation of a neuron was determined by the presence of bright synaptophysin-positive terminals; these are associated with all neurons in an unlesioned pelvic ganglion (Keast, 1995b). Similar numbers of sympathetic and parasympathetic neurons are located in this ganglion, and after either type of partial decentralization approximately half of the neurons lose their synaptophysin-positive varicosities (Keast, 1995b).

Four days after total decentralization, virtually all bright synaptophysin staining disappeared, with the exception of a very small number of baskets associated with neurons in the dorsal region of the ganglion. It is likely that these originate from enteric viscerofugal neurons, because they can be removed by cutting the rectal and penile nerves that connect with the lower bowel (Luckensmeyer and Keast, 1995, 1996). A very rare population of thin fibers with small varicosities were dimly stained for synaptophysin. The structure and staining intensity of these fibers bore no resemblance to normal preganglionic terminals, and it was assumed that they were newly formed sprouts. By $12 \mathrm{~d}$, bright synaptophysin immunoreactivity was still absent, but dimly labeled fibers had increased. Many contained VIP, NPY, or TH (Table 2).

In contrast, $12 \mathrm{~d}$ after either type of partial decentralization, numerous neurons retained bright synaptophysin-positive terminals; these are neurons that have intact spinal connections (Keast, 1995b). At this time, and for all but three neurons, sprout baskets labeled for TH, VIP, or NPY were exclusively associated with neurons that had lost their spinal innervation (i.e., in which bright synaptophysin staining was absent; Fig. $7 a, b)$. Some of these
Table 1. Chemical specificity of sprout baskets supplying pelvic ganglion cells with different target organs

\begin{tabular}{lcl} 
Target organ & VIP baskets $(\%)$ & NPY baskets $(\%)$ \\
\hline Bladder & $14.3 \pm 7.3$ & $74.4 \pm 5.4$ \\
Colon & $8.6 \pm 5.5$ & $54.2 \pm 10.5$ \\
Penis & $91.8 \pm 3.4^{*}$ & $41.5 \pm 12.2$ \\
Prostate gland & $5.2 \pm 1.5$ & $87.7 \pm 7.2$ \\
Seminal vesicles & $13.6 \pm 4.1$ & $52.8 \pm 15.3$ \\
Vas deferens & $10.1 \pm 1.2$ & $73.6 \pm 9.8$
\end{tabular}

Sprout baskets associated with neurons supplying each target organ (identified by the presence of retrograde tracer) were analyzed. Above values represent the proportion of baskets associated with neurons of similar histochemistry for each group of neurons and for each peptide. No significant differences were found between the pelvic organs for the proportion of NPY baskets associated with NPY neurons or for VIP baskets with VIP neurons, with the exception of penile VIP neurons, many of which were targeted by VIP baskets $\left({ }^{*} p<0.001\right)$.

Table 2. Percentage of VIP, NPY, or TH baskets with synaptophysin immunoreactivity

\begin{tabular}{llll} 
& VIP $(\%)$ & NPY (\%) & TH $(\%)$ \\
\hline Total decentralization & $22.0 \pm 7.2$ & $41.5 \pm 4.6$ & \\
Sympathetic decentralization & $22.2 \pm 2.7$ & & \\
Parasympathetic decentralization & $35.0 \pm 8.7$ & $69.6 \pm 7.7$ & $79.0 \pm 7.4$
\end{tabular}

Percentage of VIP, NPY, and TH baskets formed $12 \mathrm{~d}$ after partial or tota decentralization that were weakly immunoreactive for synaptophysin. Data were obtained from four animals (62-139 baskets analyzed per animal) and have been expressed as mean \pm SE. No baskets were present at this time for several categories.

sprouts possessed weak synaptophysin immunoreactivity (Table 2). In some baskets of weakly stained synaptophysin fibers, only a proportion stained for a given sprout marker (VIP, NPY, or TH). This indicates that two types of sprouts may supply some neurons. On rare occasions VIP sprouts contained bright synaptophysin immunoreactivity (Fig. $7 c, d$ ), but these neurons received no other synaptophysin-positive fibers, so they lost their spinal connection. SIF cells were also synaptophysin-immunoreactive as described previously (Keast, 1995b), and occasional groups of these cells were supplied by VIP-positive baskets (Figs. 7e,f).

\section{DISCUSSION}

The consequences of removing spinal inputs from autonomic ganglion cells have been well studied, particularly in sympathetic paravertebral ganglia (Taxi and Eugene, 1995). However, the distributed nature of the inputs to the pelvic plexus, originating from upper lumbar and sacral levels, provides a situation quite different from other ganglia. In particular, physiological studies have suggested that nerve damage at either one of these levels leads to remodelling of the intact connections within the ganglion, possibly to reestablish functional synapses (Dail et al., 1989; de Groat and Kawatani, 1989; Kihara et al., 1996). Our studies have exploited the anatomical simplicity of the rat pelvic plexus and its thorough characterization in the normal adult animal to investigate this remodelling after different types of spinal nerve injury.

We have demonstrated that there is a remarkable degree of axogenesis within the rat pelvic ganglion within a few days of removing its preganglionic inputs. This axogenesis varies considerably with the type of spinal nerve damage and appears to be derived from all ganglion cell types. Most remarkably, the new fibers that grow specifically target (or perhaps avoid) some types 

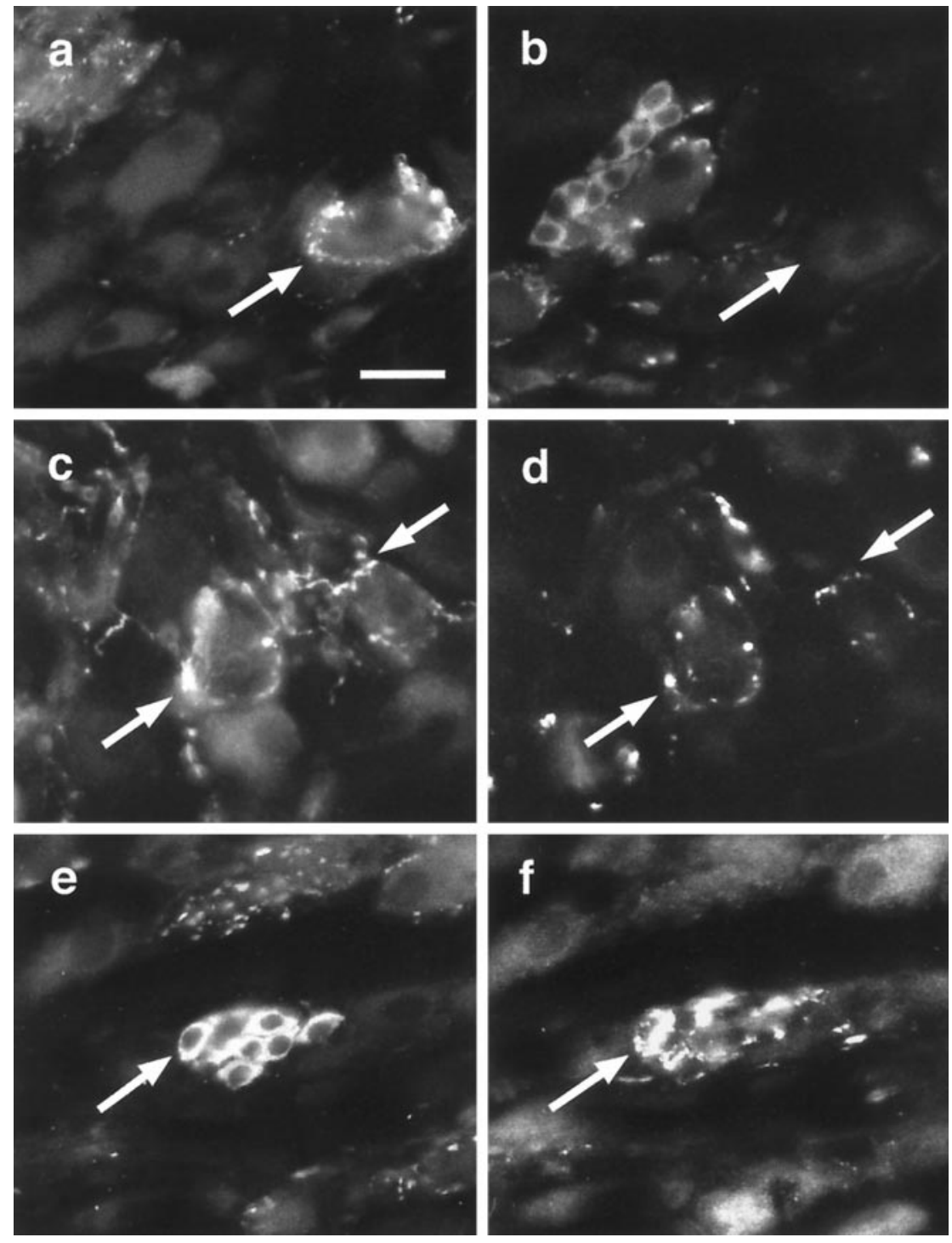

Figure 7. Synaptophysin immunoreactivity in decentralized pelvic ganglia (sacral nerve lesion, $12 \mathrm{~d}$ ). $a$, A VIP basket (arrow) supplies a decentralized neuron, as demonstrated by its lack of bright synaptophysin-positive terminals that surround nearby neurons $(b)$; SIF cells are also synaptophysin-immunoreactive. $c$, Two VIP baskets (arrows) are brightly immunoreactive for synaptophysin $(d)$, and all bright synaptophysin varicosities contain VIP. $e, A$ group of synaptophysin-immunoreactive SIF cells (arrow) are supplied by VIP sprouts $(f)$. Scale bar: $a, 25 \mu \mathrm{m}$ (refers to all micrographs). of neurons, suggesting that quite powerful and localized signaling processes exist.

Our first hypothesis was that sprouts are formed by and target specific types of neurons. This has been supported by a number of observations in both partial and total decentralization experiments. First, although all types of neurons are involved in axogenesis, each contributes to a different degree. In particular, cholinergic VIP sprouts are much more numerous than sprouts from noradrenergic NPY neurons. Second, sprouts preferentially supply neurons of dissimilar histochemistry (e.g., TH sprouts supply TH-negative neurons). Interestingly, the total sprout population does not appear to distinguish between neurons of different target organs, although to properly investigate organ-related targeting of sprouts, the cellular origin of each sprout basket would need to be identified.

Our second hypothesis was that sprouts would form after removal of only one group of spinal inputs and would supply denervated neurons. This too has been supported by our study. The strongest evidence for this comes from observations of synaptophysin immunoreactivity after nerve damage, in which neurons supplied by brightly labeled (i.e., intact) preganglionic pathways do not receive new sprouts. Instead, sprouts preferentially target neurons that have lost their spinal inputs. Although sprouts do not demonstrate immunolabeling for synaptophysin within the first few days, some slowly acquire dim staining, particularly apparent $12 \mathrm{~d}$ after nerve injury. Our studies have not tested whether this represents sufficient protein for normal synaptic function, although previous ultrastructural studies suggest that new synapses have formed in pelvic ganglia by this time (Yokota and Burnstock, 1983). The second piece of supporting evidence comes from determination of the "optimal stimulus" for sprout formation. For example, only sacral nerve damage induces noradrenergic NPY neurons to sprout axons. This type of injury does not remove the spinal connections of these neurons, because all noradrenergic NPY pelvic neurons receive lumbar inputs (Keast, 1995b). That is, sympathetic neurons with intact spinal connections sprout most profusely in a situation in which there are denervated parasympathetic neurons to innervate. It is difficult to interpret the observations of VIP sprouts, because they may originate from a mixture of sympathetic and parasympathetic neurons (Keast, 1995b).

We did not investigate whether there was any return of physiological function at times when large numbers of sprouts were present. However, it is unlikely that there was any significant 


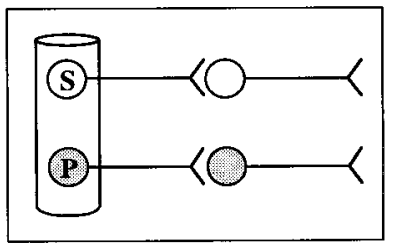

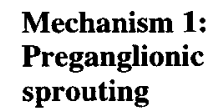

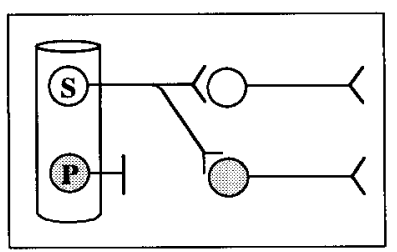

Figure 8. Proposed mechanisms of reorganization of neural pathways in the partially decentralized pelvic ganglion. The top diagram represents the intact pelvic ganglion, with equal proportions of sympathetic $(S)$ and parasympathetic $(P)$ neurons. Mechanism 1 (preganglionic sprouting) is the currently held view of how remodelling occurs in the pelvic ganglion after transection of parasympathetic preganglionic axons. Here, sympathetic preganglionic neurons form collaterals that make connections with decentralized neurons. Conversely, if sympathetic preganglionic axons were transected, parasympathetic spinal neurons would sprout collaterals to innervate denervated neurons. The present study has demonstrated that the second mechanism (postganglionic sprouting) occurs after partial decentralization. Here, postganglionic neurons with intact spinal connections sprout collaterals to supply decentralized neurons. Both mechanisms will result in activation of decentralized neurons after stimulation of the intact spinal nerves.

return of the micturition reflex, because the urinary bladder remained distended in all animals with pelvic nerve transection, up to $12 \mathrm{~d}$ after lesion. Studies in cats have shown that much longer after pelvic nerve injury (3 months), some effective transmission in bladder ganglia returns (de Groat and Kawatani, 1989). Earlier times were not tested in cats, and no studies of this type have been performed on rats. However, in peripheral circuits supplying the penis in rats, some transmission returned in pelvic ganglia after just 3 d (Dail et al., 1989). This coincides with our observations of early sprout formation. In both of the above groups of experiments, return of ganglionic transmission was tested by electrical stimulation of spinal nerves rather than by reflex activation of spinal outflow. It is therefore possible that the number or strength of new connections formed by sprouts was insufficient for reestablishing effective reflex control at times analyzed in our study. Irrespective, our observations have demonstrated that the anatomical framework required for effective communication between the CNS and the periphery is present within just a few days of spinal nerve injury.

The present study is the first to demonstrate that ganglionic connectivity can potentially be restored by a mechanism that involves the ganglion cells rather than their spinal connections (Fig. 8). Although this does not discount the possibility that intact preganglionic neurons are also involved, it adds a significant new dimension to the issue of how neural circuits respond to injury. More importantly, it expands the possible avenues by which these responses might be manipulated to maximize return of normal function. Emphasis in future studies must be placed on determining if these new connections are indeed appropriate, i.e., if they assist or impede in restoring normal reflex responsiveness. For example, unless there is concurrent reorganization of central circuitry, the new peripheral connections will not be activated under the correct circumstances (e.g., leading to an "autonomous hyperactive bladder"; de Groat and Kawatani, 1989) or will be activated along with pathways mediating antagonistic functions in the peripheral organ. It is therefore important to decide on whether the appropriate therapeutic strategy will be to enhance or discourage peripheral sprout formation.

It will also be essential to determine how these new peripheral connections impact on regeneration of damaged preganglionic terminals. This may provide new strategies for manipulating the ganglion environment to optimize synapse formation. In particular, it will be necessary to determine the relative importance of the preganglionic versus postganglionic neurons to sprout formation after partial decentralization, because each have access to different neurotrophic factors and receptors (Tuttle and Steers, 1992; Tuttle et al., 1994; Kruse et al., 1995; Steers et al., 1996; Causing et al., 1997).

The function, if any, of sprout formation after total decentralization is unknown. In our study, axogenesis appears to be transient, with decreasing levels of sprouts after $8 \mathrm{~d}$. However, longer postoperative times should be investigated to determine whether all sprouts ultimately disappear or if some are maintained, perhaps at very low density. In contrast, the present study showed that partial decentralization stimulates sprout formation that persists for at least $12 \mathrm{~d}$ and which is maintained (Dail et al., 1997) or may increase past the times analyzed. Thus, partial and total decentralization both provide a stimulus for sprout growth, but the two types of damage differ in the degree of permanence of this axogenesis.

The stimuli for sprout formation and sprout maintenance are not known. It is possible is that when peripheral organs become inactive (because of a damaged nerve supply), there is an increased secretion of neurotrophic factors, which in turn influences ganglion cell structure and connectivity. Evidence supporting this is provided by studies in rats that have undergone urethral ligation, pelvic nerve transection, or ganglionectomy. In these cases increased nerve growth factor (NGF) is synthesized by the bladder smooth muscle (Steers et al., 1991b; Tuttle et al., 1994), and sensory and motor neurons supplying the bladder increase in both size and target field (Sundin and Dahlström, 1973; de Groat et al., 1990; Steers et al., 1990, 1991a, 1992; Gabella et al., 1992; Gabella and Uvelius, 1993). It is known that cultured rat pelvic ganglion cells respond to numerous neurotrophic factors (including NGF; Tuttle and Steers, 1992; Tuttle et al., 1994; Kruse et al., 1995; Steers et al., 1996), and noradrenergic pelvic neurons in intact ganglia possess both high- and low-affinity receptors for NGF (our unpublished observations). After spinal injury, there may be a change in the exposure of pelvic ganglion cells to this and other growth factors or in their expression of growth factor receptors. This may provide a mechanism for sprout formation and targeting.

Finally, the decentralization procedures will cause axotomy of a small population of pelvic ganglion cells that project out of the hypogastric and pelvic nerves (Hulsebosch and Coggeshall, 1982; Dalsgaard et al., 1983; McLachlan, 1985; Dail and Minorsky, 1986; Tabatabai et al., 1986; Baron and Jänig, 1988). Although it is possible that these damaged neurons also produce sprouts, there are very few of these neurons ( 20-60, Dail and Minorsky, 1986; our unpublished observations), so they are unlikely to account for the large numbers of fibers produced in pelvic ganglia after spinal nerve damage.

In conclusion, we have demonstrated that a rapid and selective 
axogenesis occurs within the pelvic ganglion after spinal nerve injury. This substantial remodelling of peripheral circuitry could impact significantly on the restoration of peripheral motor outflow to numerous pelvic organs if central circuits are reestablished appropriately. Clearly, it is important to optimize the regeneration of spinal nerves to innervate their ganglion cells. However, the additional contribution made by ganglion cell axogenesis may provide novel strategies by which these pathways can be experimentally manipulated, to facilitate more rapid return of appropriate peripheral connectivity.

\section{REFERENCES}

Baron R, Jänig W (1988) Neurons projecting rostrally in the hypogastric nerve of the cat. J Auton Nerv Syst 24:81-86.

Causing CG, Gloster A, Aloyz R, Bamji SX, Chang E, Fawcett J, Kuchel G, Miller FD (1997) Synaptic innervation density is regulated by neuron-derived BDNF. Neuron 18:257-267.

Dail WG, Evan AP (1978) Effects of chronic deafferentation on adrenergic ganglion cells and small intensely fluorescent cells. J Neurocytol 7:25-37.

Dail WG, Minorsky N (1986) Composition of the pelvic nerve. Exp Neurol 92:278-283.

Dail WG, Moll MA, Weber K (1983) Localization of vasoactive intestinal polypeptide in penile erectile tissue and in the major pelvic ganglion of the rat. Neuroscience 10:1379-1386.

Dail WG, Walton G, Olmsted MP (1989) Penile erection in the rat: stimulation of the hypogastric nerve elicits increases in penile pressure after chronic interruption of the sacral parasympathetic outflow. J Auton Nerv Syst 28:251-258.

Dail WG, Galindo R, Leyba L, Barba V (1997) Denervation-induced changes in perineuronal plexuses in the major pelvic ganglion of the rat: immunohistochemistry for vasoactive intestinal polypeptide and tyrosine hydroxylase and histochemistry for NADPH-diaphorase. Cell Tissue Res 287:315-324.

Dalsgaard C-J, Hökfelt T, Schultzberg M (1983) Origin of peptidecontaining fibers in the inferior mesenteric ganglion of the guinea-pig: immunohistochemical studies with antisera to substance P, enkephalin, vasoactive intestinal peptide, cholecystokinin, and bombesin. Neuroscience 9:191-211.

de Groat WC, Kawatani M (1989) Reorganization of sympathetic preganglionic connections in cat bladder ganglia following parasympathetic denervation. J Physiol (Lond) 409:431-449.

de Groat WC, Kawatani M, Hisamitsu T, Cheng C, Ma C, Thor K, Steers W, Roppolo JR (1990) Mechanisms underlying the recovery of urinary bladder function following spinal cord injury. J Auton Nerv Syst 30:S71-S78.

Gabella G, Uvelius B (1993) Effect of decentralization or contralateral ganglionectomy on obstruction-induced hypertrophy of rat urinary bladder muscle and pelvic ganglion. J Neurocytol 22:827-834.

Gabella G, Berggren T, Uvelius B (1992) Hypertrophy and reversal of hypertrophy in rat pelvic ganglion neurons. J Neurocytol 21:649-662.

Hulsebosch CE, Coggeshall RE (1982) An analysis of the axon populations in the nerves to the pelvic viscera in the rat. J Comp Neurol 211:1-10.

Johnson DA (1988) Regulation of intraganglionic synapses among rabbit parasympathetic neurones. J Physiol (Lond) 397:51-62.

Keast JR (1991) Patterns of co-existence of peptides and differences of nerve fibre types associated with noradrenergic and non-noradrenergic (putative cholinergic) neurons in the major pelvic ganglion of the male rat. Cell Tissue Res 266:405-415.

Keast JR (1992) Location and peptide content of pelvic neurons supplying the muscle and lamina propria of the rat vas deferens. J Auton Nerv Syst 40:1-12.

Keast JR (1995a) Pelvic ganglia. In: Autonomic ganglia (McLachlan EM, ed), pp 445-480. Chur, Switzerland: Harwood Academic.

Keast JR (1995b) Visualisation and immunohistochemical characterization of sympathetic and parasympathetic neurons in the male rat major pelvic ganglion. Neuroscience 66:655-662.
Keast JR, de Groat WC (1989) Immunohistochemical characterisation of pelvic neurons which project to the bladder, colon, or penis in rats. J Comp Neurol 288:387-400.

Kepper M, Keast J (1995) Immunohistochemical properties and spinal connections of pelvic autonomic neurons that innervate the rat prostate gland. Cell Tissue Res 281:533-542.

Kepper ME, Keast JR (1997) Location, immunohistochemical features, and spinal connections of autonomic neurons innervating the rat seminal vesicles. Biol Reprod 57:1164-1174.

Kihara K, Kakizaki H, de Groat WC (1996) Reorganisation of the innervation of the vas deferens after sympathetic decentralization Am J Physiol 274:R1481-R1488.

Kruse MN, Bray LA, de Groat WC (1995) Influence of spinal cord injury on the morphology of bladder afferent and efferent neurons. J Auton Nerv Syst 54:215-224.

Luckensmeyer GB, Keast JR (1995) Immunohistochemical characterisation of sympathetic and parasympathetic pelvic neurons projecting to the distal colon in the male rat. Cell Tissue Res 281:551-559.

Luckensmeyer GB, Keast JR (1996) Immunohistochemical characterisation of viscerof ugal neurons projecting to the inferior mesenteric and major pelvic ganglia in the male rat. J Auton Nerv Syst 61:6-16.

McLachlan EM (1985) The components of the hypogastric nerve in male and female guinea pigs. J Auton Nerv Syst 13:327-342.

Minorsky NM, Dail WG (1993) The effect of chronic decentralization on the enkephalin immunoreactive plexus around penile ganglionic neurons. J Auton Nerv Syst 45:215-223.

Quillam JP, Tamarind DL (1972) Electron microscopy of degenerative changes in decentralised rat superior cervical ganglia. Micron $3: 454-472$.

Ramsay DA, Matthews MR (1985) Denervation-induced formation of adrenergic synapses in the superior cervical sympathetic ganglion of the rat and the enhancement of this effect by postganglionic axotomy. Neuroscience 16:997-1026.

Sargent PB, Dennis MJ (1977) Formation of synapses between parasympathetic neurones deprived of preganglionic innervation. Nature 268:456-458.

Steers WD, Ciambotti J, Erdman S, de Groat WC (1990) Morphological plasticity in efferent pathways to the urinary bladder of the rat following urethral obstruction. J Neurosci 10:1943-1951.

Steers WD, Ciambotti J, Etzel B, Erdman S, de Groat WC (1991a) Alterations in afferent pathways from the urinary bladder of the rat in response to partial urethral obstruction. J Comp Neurol 310:401-410.

Steers WD, Kolbeck S, Creedon DJ (1991b) Nerve growth factor in the urinary bladder of the adult regulates neuronal form and function. J Clin Invest 88:1709-1715.

Steers WD, Ciambotti J, Etzel J, Erdman S, de Groat WC (1992) Alterations in the afferent pathways from the urinary bladder of the rat in response to partial urethral obstruction. J Comp Neurol 310:401-410

Steers WD, Creedon DJ, Tuttle JB (1996) Immunity to nerve growth factor prevents afferent plasticity following urinary bladder hypertrophy. J Urol 155:379-385.

Sundin T, Dahlström A (1973) The sympathetic innervation of the urinary bladder and urethra in the normal state and after parasympathetic denervation at the spinal root level. Scand J Urol Nephrol 7:131-149.

Tabatabai M, Booth AM, de Groat WC (1986) Morphological and electrophysiological properties of pelvic ganglion cells in the rat. Brain Res 382:61-70.

Taxi J, Eugène D (1995) Effects of axotomy, deafferentation, and reinnervation on sympathetic ganglionic synapses: a comparative study. Int Rev Cytol 159:195-263.

Tuttle JB, Steers WD (1992) Nerve growth factor responsiveness of cultured major pelvic ganglion neurons from the adult rat. Brain Res 588:29-40.

Tuttle JB, Steers WD, Albo M, Nataluk E (1994) Neural input regulates tissue NGF and growth of the adult rat urinary bladder. J Auton Nerv Syst 49:147-158.

Yokota R, Burnstock G (1983) Decentralisation of neurones in the pelvic ganglion of the guinea-pig: reinnervation by adrenergic nerves. Cell Tissue Res 232:399-411. 